$\underline{\text { Supporting Information }}$

\title{
Delineating Heme-Mediated versus Direct Protein Oxidation in Peroxidase-Activated Cytochrome $c$ by Top-Down Mass Spectrometry
}

\author{
Victor Yin, Derek Holzscherer, and Lars Konermann* \\ Department of Chemistry, The University of Western Ontario, London, Ontario, \\ N6A 5B7, Canada
}

This file contains:

Figure S1. Structure of CT.

Figure S2. UV/vis absorption spectra of cyt $c$ before and after heme removal.

Figure S3. Mass spectra of DTT-treated aposs-cyt $c$.

Figure S4. Far-UV CD spectra of cyt $c$.

Figure S5. Top-down CID-IM-MS of CT-holo-cyt $c$.

Figure S6. Top-down CID-IM-MS of GRT-labeled CT-holo-cyt $c$.

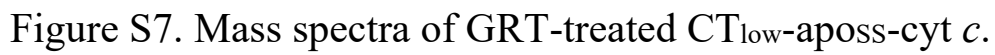

Figure S8. Bottom-up MS/MS of oxidized Trp peptides.

Figure S9. Mass spectra of GRT-labeled MP11/CTlow-aposs-cyt $c$. 


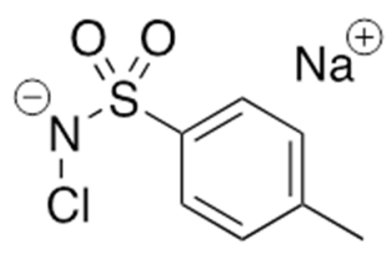

Figure S1: Structure of the oxidant chloramine-T (N-chloro-4-toluosulfonamide), referred to as "CT" in this work. The key CT-induced oxidative modifications examined in this work are Met $\rightarrow$ MetO (sulfoxide formation, +16 Da) and Lys- $\mathrm{CH}_{2}-\mathrm{NH}_{2} \rightarrow$ Lys-CHO ("carbonylation", $-1 \mathrm{Da}$ ). 


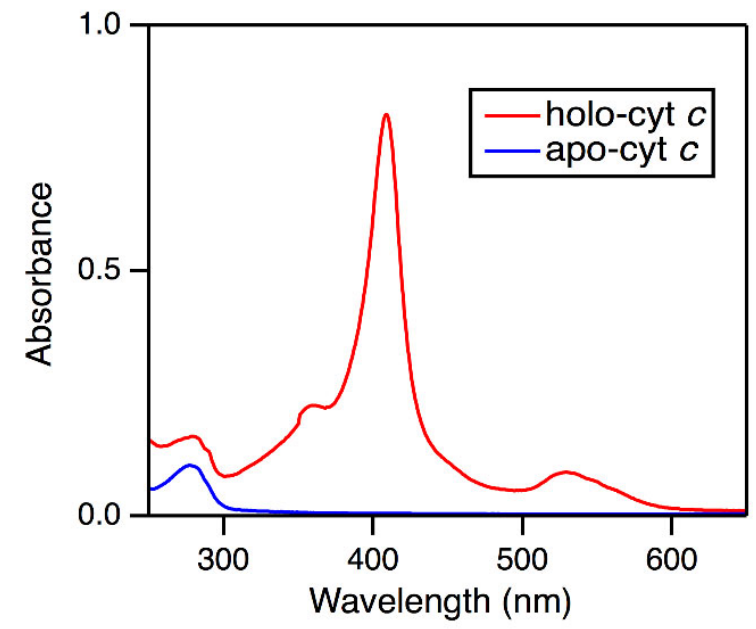

Figure S2. UV/Vis absorption spectra of cyt $c$ before and after heme removal: holo-cyt $c$ (red), apo-cyt $c$ (blue). 


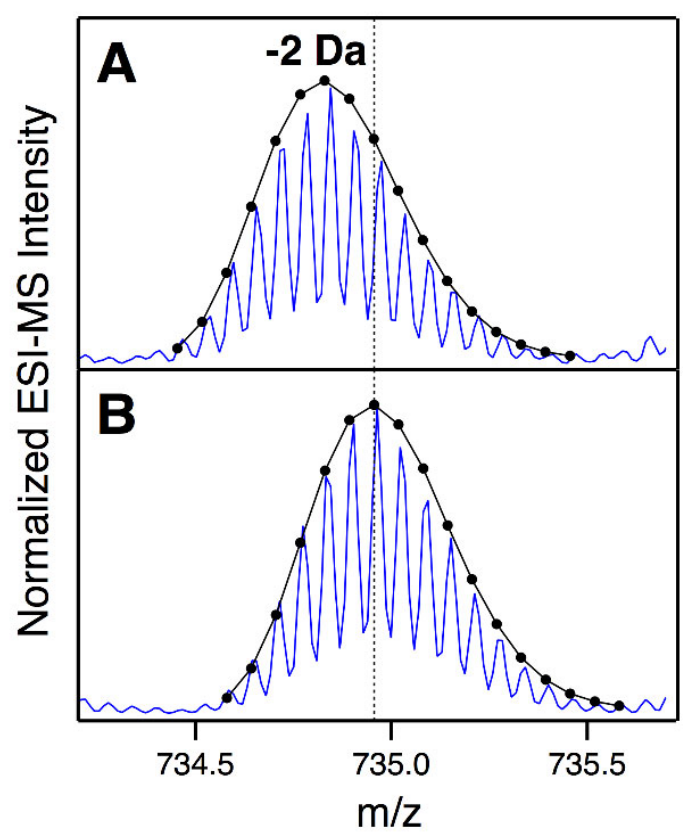

Figure S3. Mass spectrum (charge state 16+) of aposs-cyt $c$ (A) before DTT treatment and (B) following DTT treatment. The vertical dashed line is included as a visual aid. Following DTT treatment, the -2 Da mass shift is lost, and the original (free $-\mathrm{SH}$ ) mass is recovered. 


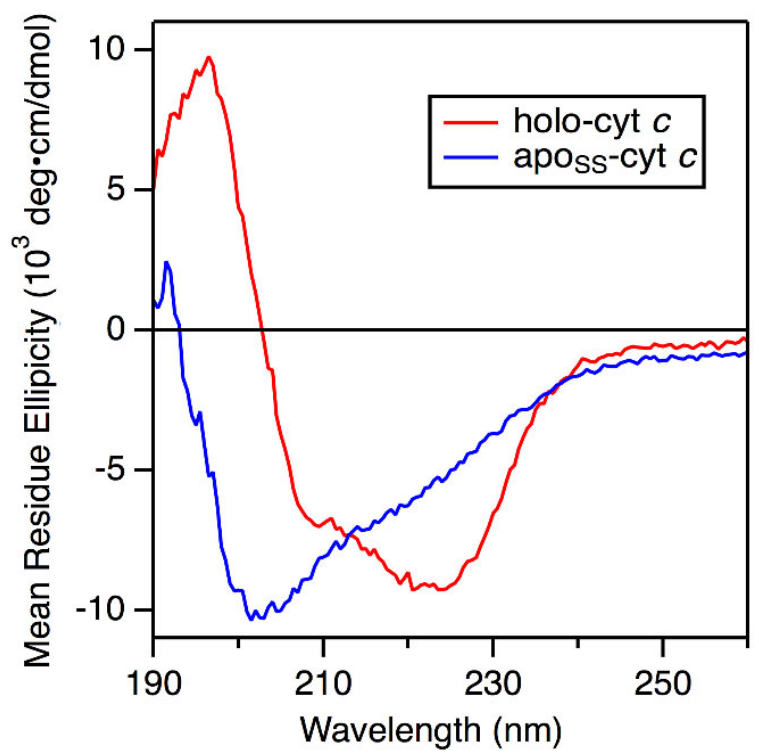

Figure S4. Far-UV CD spectra of holo-cyt $c$ (red) and aposs-cyt $c$ (blue). The minimum close to $200 \mathrm{~nm}$ for aposs-cyt $c$ reveals that heme removal causes the protein to adopt an extensively unfolded conformation. 


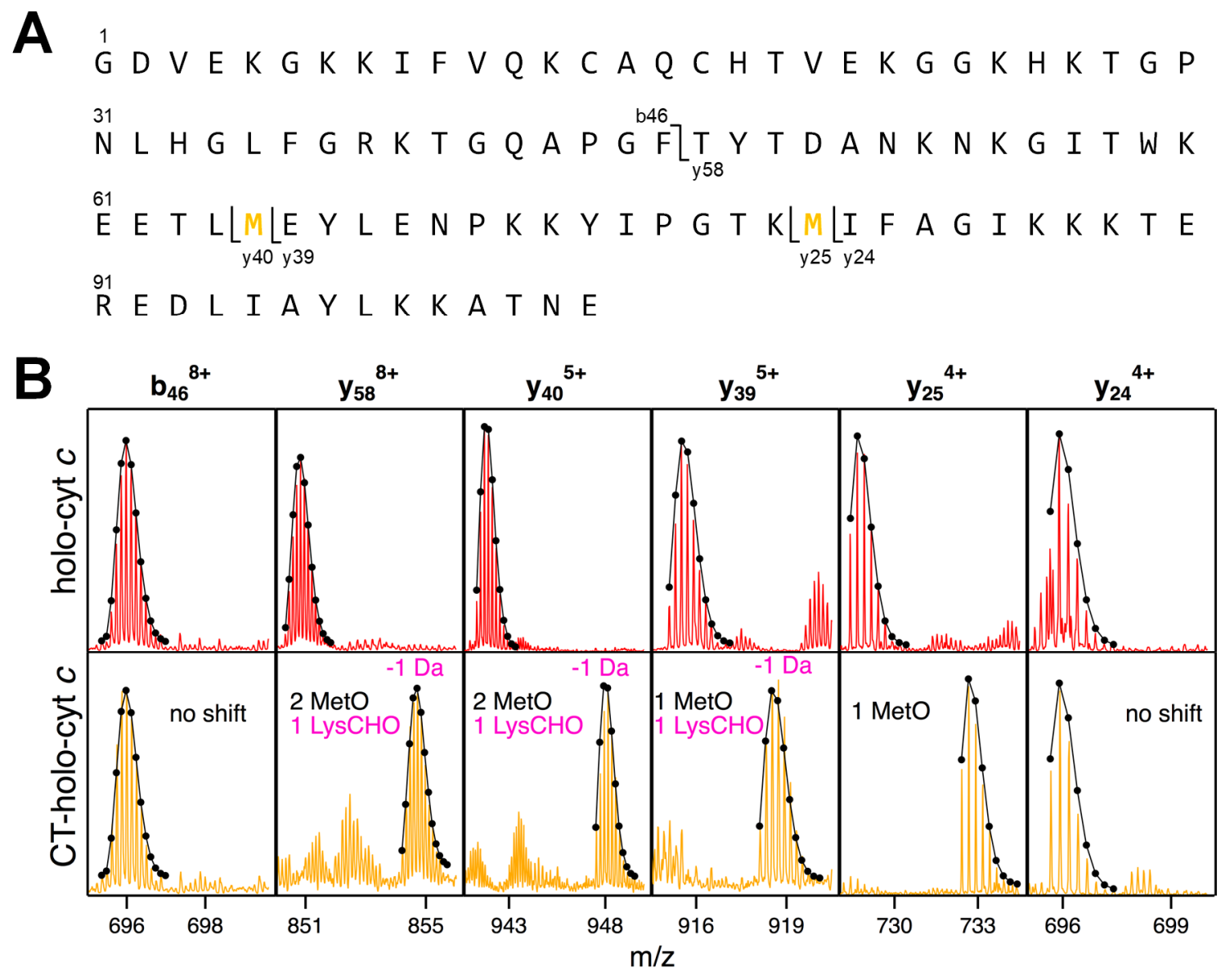

Figure S5. Top-down CID-IM-MS analysis of CT-holo-cyt $c$. (A) protein sequence with selected fragmentation sites. The two modified Met residues (MetO sites) are highlighted in color. (B) Representative top-down CID-IM-MS fragment ions. Top: holo-cyt $c$ (untreated control). Bottom: CT-holo-cyt $c$. Each spectrum is overlaid with its corresponding theoretical isotopic envelope (solid circles and black lines). 


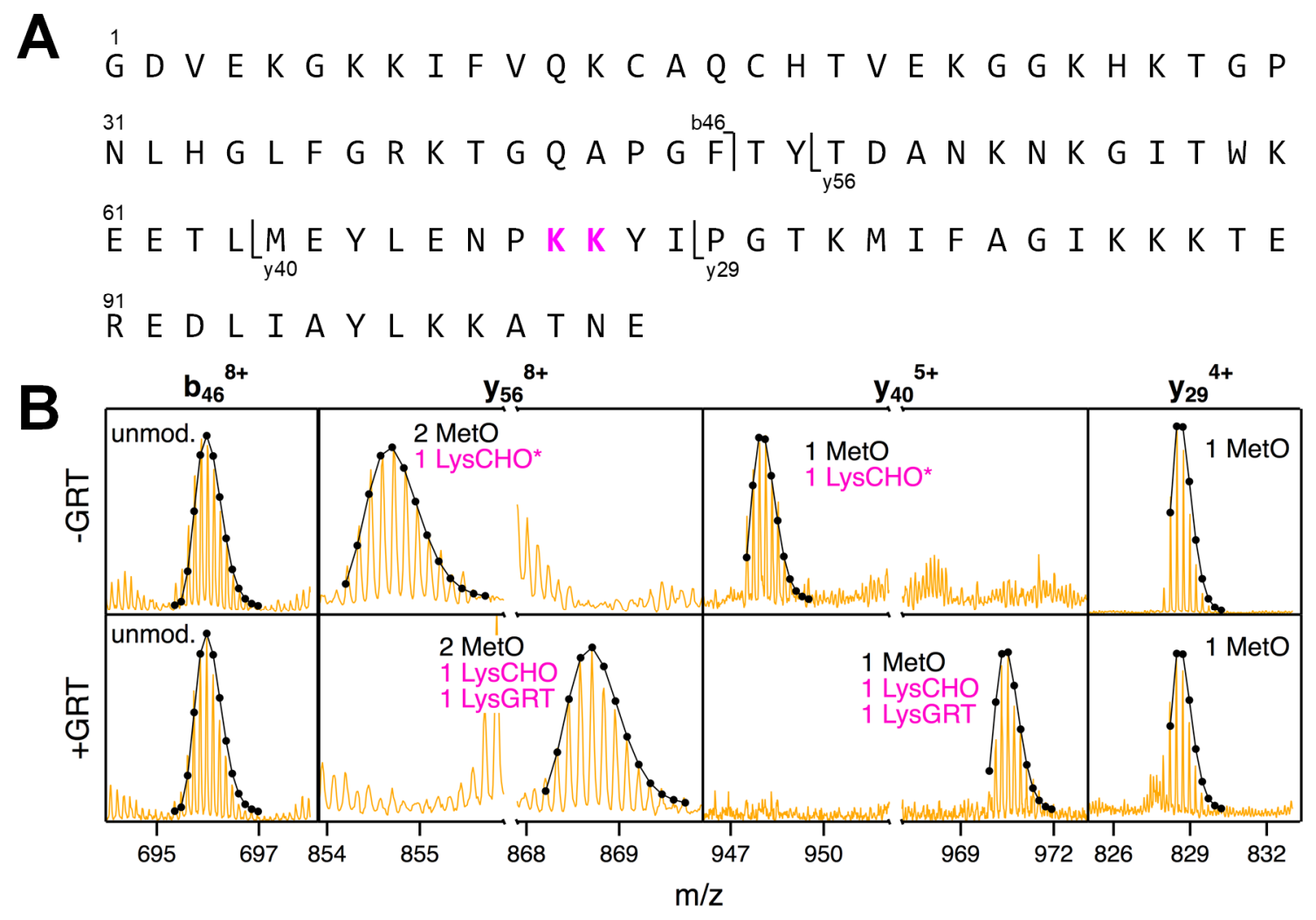

Figure S6. Top-down CID-IM-MS analysis of GRT-labeled CT-holo-cyt $c$. (A) protein sequence with selected fragmentation sites. The two modified Lys residues are highlighted in colour. (B) Representative top-down CID-IM-MS fragment ions. Top: CT-holo-cyt $c$ (no GRT control). Bottom: GRT-labeled CT-holo-cyt $c$, using the [M+2 O + 1 LysCHO + 1 GRT] peak as precursor. Each spectrum is overlaid with its corresponding theoretical isotopic envelope (solid circles and black lines). 


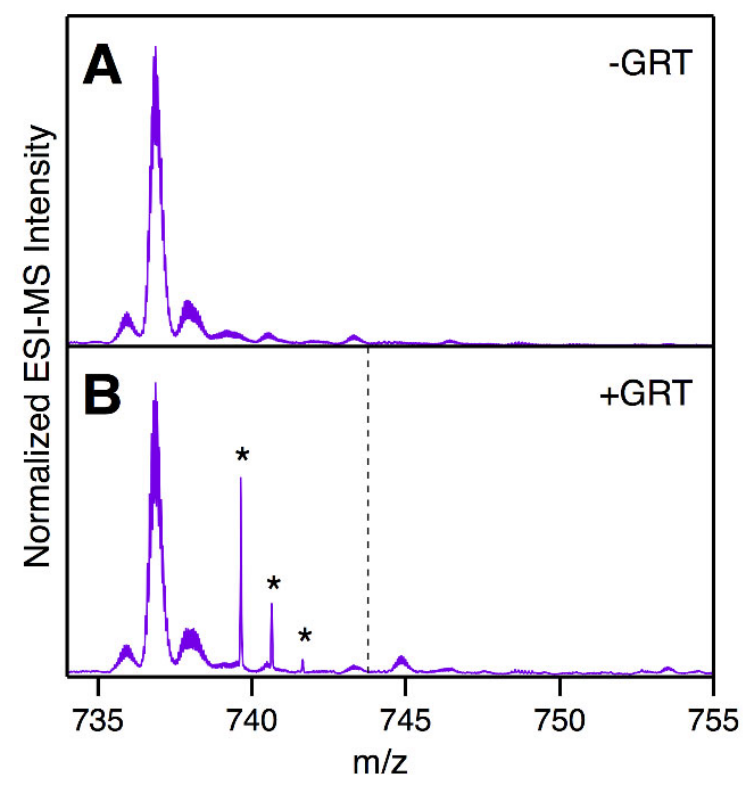

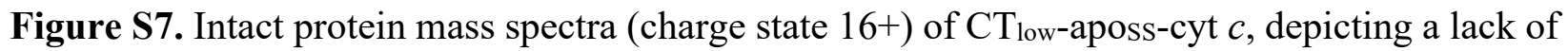
GRT labeling due to the absence of LysCHO. (A) Spectrum before GRT exposure. (B) Spectrum after GRT exposure. The vertical line in panel B marks position where the GRT-labeled protein would be expected. The asterisks mark background contaminant signals. 


$$
\text { G I T }\left\lfloor\underline{\underline{W}}\left|\frac{\mathrm{W}}{y_{2}}\right| K_{y_{1}}\right.
$$

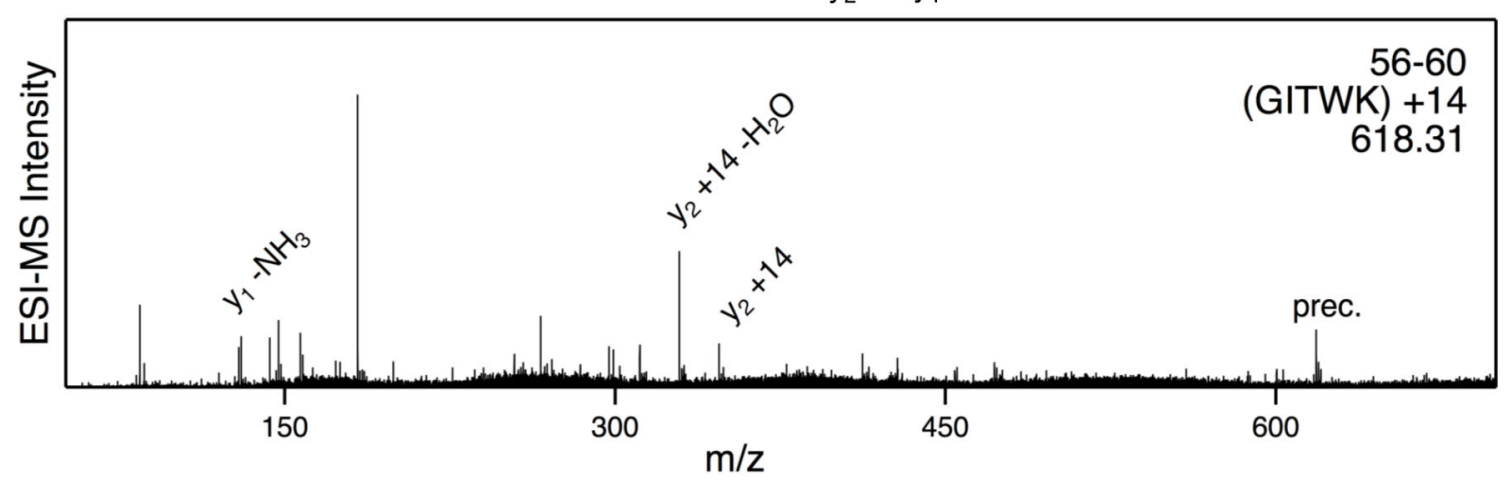

Figure S8. Bottom-up MS/MS of the tryptic peptide ${ }^{56} \mathrm{GITWK}^{60}$ depicting oxidation of Trp59 $(+14 \mathrm{Da})$ in CT-aposs-cyt $c$. This peptide was not detected in any other sample. 

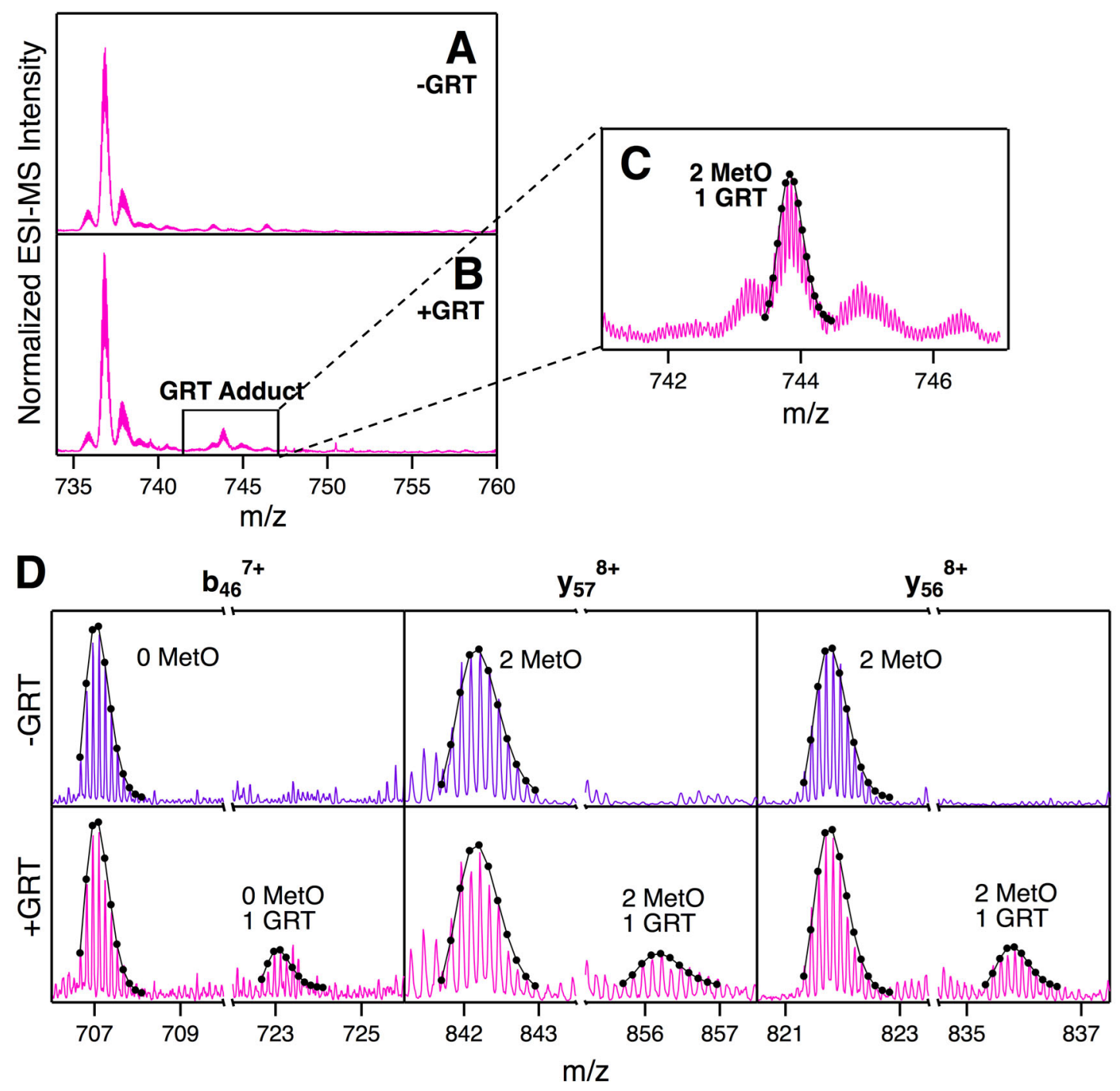

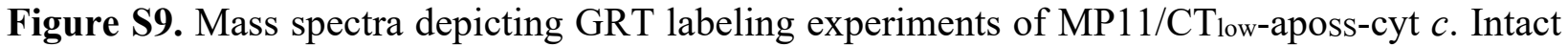
protein mass spectra (charge state $16+$ ) are depicted in panels A-C. (A) MP11/CT low-aposs-cyt $c$ without GRT, and (B) with GRT. (C) Magnified view of (B) highlighting the GRT-labeled protein, overlaid with its simulated isotope distribution. (D) Representative top-down CID-IM-MS fragment ions, highlighting the presence of multiple ion populations in GRT-labeled MP11/CTlowaposs-cyt $c$. Top: CT low-apossCyt $c$ (no GRT treatment). Bottom: GRT-labeled MP11/CTlowaposs Cyt $c$. The b46 and y57/56 ions shown in panel D cover mutually exclusive regions of the protein. If the precursor ion were homogeneously modified at a single site, then only one of these complementary fragment ions could contain the modification. The observation of a GRTcontaining peak in both fragment ions demonstrates that there is more than one GRT site. 